\title{
The Origin of Gravitation and Electromagnetism
}

\author{
Zhong-Cheng Liang \\ School of Electronic and Optical Engineering, Nanjing University of Posts and Telecommunications, Nanjing, China \\ Email: zcliang@njupt.edu.cn
}

\begin{abstract}
Assuming that protons and electrons are three-dimensional body particles that have only mass and volume, the volume repulsion can ensure the finiteness of particle density. A scalar potential and a vector potential are constructed respectively with the mass density and the momentum density. A set of particle field equations is derived from the scalar and vector potentials with the help of vector calculus. The equation set is general field equations in three-dimensional flat space, instead of that in four-dimensional curved spacetime. It is shown that the particle field includes gradient field, divergence field and curl field. The gradient field predicts both attraction and repulsion between particles, the divergence field represents the undulation of particles, and the curl field describes the vortex motion of the particles. It is proven that gravitation and electromagnetism both originate from the interaction of body particles. The undulation of pure electronic system represents both gravitational waves and electromagnetic waves. If graviton and photon are regarded as body particles identical to electron, the electrons are the dark matter in the background of the universe.
\end{abstract}

Keywords: Particle, field, interaction, gravitation, electromagnetism

\section{Introduction}

In 1687, Newton put forward the laws of motion and the law of universal gravitation, laid the foundation of classical mechanics [1]. The gravitational force is directly proportional to the mass of object, and is inversely proportional to the square of the distance between objects. In 1865, Maxwell proposed the electromagnetic field equations on the basis of his predecessors' work, which laid the foundation of classical electrodynamics [2]. The electric (magnetic) force is directly proportional to the charge (current) and inversely proportional to the square of the distance between charges (current elements). Despite of this similarity, gravitation and electromagnetism are regarded as different fundamental interactions because of the particularity of magnetic force. In classical physics, the gravitational force is thought to be transmitting through vacuum instantaneously, while the electromagnetic force is considered to be transmitting through electromagnetic field at a limited speed of light.

In order to maintain the motion invariance of electromagnetic laws, Einstein put forward the special theory of relativity in 1905 and established the conception that electromagnetism is related to the nature of time and space [3]. In his general theory of relativity, he further interpreted the origin of gravitation as the bending of space-time [4]. On the other hand, quantum field theory treats particles as excited states of their underlying fields. The origin of electromagnetic force is attributed to the exchange of photons. As messenger particles, photons are point particles that have no mass and volume. The background of quantum field theory is Minkowski's flat spacetime, the final outcome is that tens of fundamental particles are needed to explain the electromagnetic, strong and weak interactions in the Standard Model of particle physics [5]. A number of quantum gravity theories are proposed to deal with the problem of gravitation, which explain the origin of gravity by the exchange of gravitons between objects [6]. One of them is the string theory developed from quantum field theory. The unusual aspect of string theory is to expand particles from zero-dimensional points to one-dimensional strings [7]. The success of string theory is in that one mode will alwavs correspond to a graviton. However, the price of this success are unusual features such as six extra dimensions of space in addition to the usual three for space and one for time. Currently, there is still no complete and consistent quantum theory of gravity. Apart from the major formal and conceptual problems that need to be overcome, the common problem they encounter is that so far there is no way to put quantum gravity predictions to experimental verification.

Theoretical physics is logic formal system based on the core concepts of space, time, particle, field and interactions. According to the set theory [8], logic formal systems based on different conceptual sets are not 
equivalent to each other. According to Gödel's incompleteness theorem, a formal axiomatic system is not complete even if it is self-consistent [9]. In fact, current physics is neither complete nor consistent. The origins of gravitation and electromagnetism are major problems that have not been fundamentally solved.

Three years ago, we proposed a theory of particle field based on the model of three-dimensional body particle that has only mass and volume [10]. The basic idea is to introduce a vector potential in addition to the scalar gravitational potential, and then to derive a complete set of particle field equations. The particle field theory is established within the background of three-dimensional Euclidian space, which belongs to the category of classical field theory $[10,11]$. The breakthrough lies in the modification of the particle model, which expands the particles from zero-dimensional point to three-dimensional body. After introducing the particle field theory, this article gives a unified explanation to the origin of gravitation and electromagnetism.

\section{Basic Concepts and Principles}

\subsection{Principle of Object Structure}

\subsubsection{Concepts and axioms}

(a) Object. An object is three-dimensional body that has only two radical attributes: mass and volume.

(b) Particle. A particle is three-dimensional body that has only two radical attributes: mass and volume. Such particle is called body particle (or entity particle [11]) in contrast with the point particle.

(c) Axiom of object structure (Axiom 1). Any object is composed of particles with nesting structure. An object can be decomposed into discrete and finite particles.

(d) Primary particle: A primary particle is indivisible particle that has constant mass.

(e) Axiom of primary particle (Axiom 2). There are only two types of primary particles of different mass: proton and electron.

(f) Theorem of mass imperishability. Mass is an inherent property of particles, and the mass of a primary particle is constant. All objects are made up of primary particles, so the mass of an object cannot be annihilated.

\subsubsection{Primary particles}

In the international system of units (SI system), the mass of proton is $M_{p}=1.6726216 \times 10^{-27} \mathrm{~kg}$, the mass of electron is $M_{e}=9.1093821 \times 10^{-31} \mathrm{~kg}$. Mass and volume are the radical attributes of primary particles. Obviously, spin and deformation are their motion characteristics. This article will prove that electric charge is an attribute equivalent to the mass of particles. Therefore, the proton and electron defined in this article are the same things as those in modern physics.

A neutron can decay into proton and electron, which indicates that neutron is composed of proton and electron. During beta decay, spin and deformation can take away some energy. It is unnecessary to assume the massless neutrinos. The decomposition of proton and electron has not been observed so far, therefore, proton and electron are the only primary particles.

\subsubsection{Object structure}

If electron is represented by symbol 0 and proton by symbol 1, any object can be represented by a set consisting of 0 and 1 . (In this article, set symbols are represented by double-line font.)

(a) Electron. The set of $a$ electrons is represented as

$$
\mathbb{E}^{a}=\underbrace{\{0,0,0, \cdots, 0\}}_{a} .
$$

(b) Proton. The set of $b$ protons is represented as

$$
\mathbb{P}^{b}=\underbrace{\{1,1,1, \cdots, 1\}}_{b} .
$$

(c) Neutron. Neutron is a composite particle represented by $\mathbb{N}=\{0,1\}$. The mass of neutron is $M_{p}+M_{e}$. The set of $c$ neutrons is represented as

$$
\mathbb{N}^{c}=\underbrace{\{\{0,1\},\{0,1\},\{0,1\}, \cdots,\{0,1\}\}}_{c} .
$$

(d) Atomic nucleus. An atomic nucleus contains $b$ protons and $c$ neutrons,

$$
\mathbb{N} \text { uncleus }(b, c)=\mathbb{P}^{b} \cup \mathbb{N}^{c}=\left\{\mathbb{P}^{b}, \mathbb{N}^{c}\right\} \text {. }
$$


Atomic nucleus contains primary particles $b+2 c$ and has mass $(b+c) M_{p}+c M_{e}$.

(e) Ion. An ion is made up of a nucleus and the electrons outside the nucleus, which is represented as

$$
\mathbb{I}^{Z_{I}}(a, b, c)=\mathbb{E}^{a} \cup \mathbb{N} \text { undeus }(b, c)=\left\{\mathbb{E}^{a},\left\{\mathbb{P}^{b}, \mathbb{N}^{c}\right\}\right\},
$$

where $a$ is the number of electrons outside the nucleus, $b$ is the number of protons (atomic number) and $c$ is the number of neutrons in the nucleus. $Z_{I}=b-a$ is the number of ionic charges. An ion contains primary particles $N_{I}=a+b+2 c$ and has mass $M_{I}=(b+c) M_{p}+(a+c) M_{e}$.

(f) Atom. Atom is a combination of the atomic core and the valence electrons, which is represented as

$$
\operatorname{Art} \mathbb{A m}(b, c)=\left\{\mathbb{E}^{a_{1}},\left\{\mathbb{E}^{a_{0}},\left\{\mathbb{P}^{b}, \mathbb{N}^{c}\right\}\right\},\right.
$$

where $\mathbb{E}^{a_{1}}$ is valence electron. $a_{0}+a_{1}=b$ is atomic number, and $c$ stands for different isotopes. For example,

$$
\mathbb{H}(c)=\left\{\mathbb{E}^{0},\left\{\mathbb{E}^{1},\left\{\mathbb{P}^{1}, \mathbb{N}^{c}\right\}\right\}\right\}
$$

represents the atom of hydrogen. $\mathbb{H}(0)$ is protium, $\mathbb{H}(1)$ is deuterium and $\mathbb{H}(2)$ is tritium. An atom contains primary particles $N_{A}=2(b+c)$ and has mass $M_{A}=(b+c)\left(M_{p}+M_{e}\right)$.

(g) Molecule. A molecule is a composite particle made up of ions. If the molecule is made up of $m$ ions, then the molecule $\mathbb{M}^{Z_{M}}$ is represented as

$$
\mathbb{M}^{Z_{M}}=\left\{\mathbb{I}_{1}^{Z_{I 1}}, \mathbb{I}_{2}^{Z_{I 2}}, \cdots, \mathbb{I}_{k}^{Z_{I k}}, \cdots, \mathbb{I}_{m}^{Z_{I m}}\right\},
$$

where $Z_{M}=\sum_{k=1}^{m} Z_{I k}$ is the charge number of the molecule. A molecule contains primary particles $N_{M}=\sum_{k=1}^{m} N_{I k}$ and has mass $M_{M}=\sum_{k=1}^{m} M_{I k}$.

(h) Molecular cluster. The upper level structure made up of molecules is called molecular cluster. If the molecular cluster consists of $n$ molecules, then the molecular cluster $\mathbb{C}^{Z_{C}}$ is a set of molecules

$$
\mathbb{C}^{Z_{C}}=\left\{\mathbb{M}_{1}^{Z_{M 1}}, \mathbb{M}_{2}^{Z_{M 2}}, \cdots, \mathbb{M}_{k}^{Z_{M k}}, \cdots, \mathbb{M}_{n}^{Z_{M n}}\right\},
$$

where $Z_{C}=\sum_{k=1}^{n} Z_{M k}$ is the charge number of the molecular cluster. A molecular cluster contains primary particles $N_{C}=\sum_{k=1}^{n} N_{M k}$ and has mass $M_{C}=\sum_{k=1}^{n} M_{M k}$.

Particles of higher level structure can be described in a similar way. According to above reasoning, all objects are composed of electrons and protons with nesting structure. Any object contains finite particles and has definite mass, just like a piece of information in the electronic computer is a set of strings consisting finite bits of 0 and 1.

\subsubsection{Particle system}

Any object can be regarded as a system of body particles. If $P_{i}$ represents the $i$-th particle, the object can be represented by

$$
\mathbb{O} \text { bject }=\left\{P_{i} \mid i=1,2,3, \cdots, N\right\}=\left\{P_{i}\right\},
$$

$\left\{P_{i}\right\}$ is a simplified form for particle system with the convention of $i=1,2,3, \cdots, N$.

Let $M_{i}$ be the mass of $P_{i}$, the total mass of the object can be expressed by

$$
M=\sum_{i=1}^{N} M_{i}=N \cdot \sum_{i=1}^{N}\left(\frac{M_{i}}{N}\right) \equiv N \cdot \bar{M}, \quad \bar{M}=\sum_{i=1}^{N}\left(\frac{M_{i}}{N}\right),
$$

where $\bar{M}$ is the average mass of particles. The symbol " $\equiv$ " is used as both identity sign and definition sign in this article.

\subsubsection{Object density}

An object contains $N$ particles, has mass $M$ and volume $V$, then we can define the particle density $n$ and the mass density $\rho$ as

$$
n \equiv N / V, \quad \rho \equiv M / V .
$$

The definition of body particle ensures that the density is finite, and the infinity of the density is ruled out from principle.

\subsection{Principles of Space, Time and Motion}

\subsubsection{Concepts and axioms}

(a) Space. Space is the place in which the objects exist and move. The real space is continuous, uniform and three-dimensional.

(b) Axiom of real space (Axiom 3). Real space is filled with particles. There exists no empty space that 
has no particles.

(c) Time. Time is a progression with which the objects exist and move. The time is continuous, uniform and unidirectional.

(d) Motion. Motion is the process that the state of object changes with time in space.

(e) Volume. The volume of an object is the space required for the motion of its internal particles.

(f) Theorem of volume repulsion. The motion of particles requires space and time. The volume of different particles does not intersect each other in real space at the same time.

(g) Real physics. Real physics is a theory based on real space and restricted in the domain (field) of real number.

\subsubsection{Space and position}

The real space is represented by the position $P(\boldsymbol{r})$ where $\boldsymbol{r}$ is a three-dimensional vector called position vector. Specifying a reference point $O$, called origin, the position vector is a line segment directing from the origin $O$ to the point $P$. The fact that the origin can be chosen arbitrarily by observer is called origin subjectivity. In Cartesian coordinate, the position vector is expressed as

$$
\boldsymbol{r} \equiv \overrightarrow{O P}=(x, y, z)=x \mathbf{i}+y \mathbf{j}+z \mathbf{k},
$$

where $x, y, z$ are Cartesian components of the position vector, and $\mathbf{i}, \mathbf{j}, \mathbf{k}$ are the unit vectors in the direction of the coordinate axis.

The length of position vector is the distance between $O$ and $P$,

$$
r=|\boldsymbol{r}|=|\overrightarrow{O P}|=\sqrt{x^{2}+y^{2}+z^{2}} .
$$

Let $r_{s}$ be the scale of space, the length of position vector can be expressed by

$$
r=r_{s} \cdot \tilde{r},
$$

where $\tilde{r}$ is the digit of length. The space scale $r_{s}$ is the unit of length, which is termed spacon.

\subsubsection{Time and synchronization}

Let $t_{s}$ be the scale of time, the time is defined as

$$
t \equiv t_{s} \cdot \tilde{t} ; \quad \tilde{t}=0,1,2, \cdots, k, \cdots, N_{t} .
$$

The scale of time $t_{s}$ is the unit of time, which is termed timon. $\tilde{t}$ is the digit of time, which is a sequence of natural numbers. $\tilde{t}=0$ is the starting time, $\tilde{t}=N_{t}$ is the ending time, and $\tilde{t}=k$ is any time.

Synchronization is a protocol to set a starting time for all spatial positions. It is implemented by a communication method described as follows. A signal $t=0$ is sent from the origin $O$ with a communication speed $c$. When the observer at $P(\boldsymbol{r})$ receives the signal, the time is set to $t=r / c$. For example, if the Greenwich is taken as the origin and the speed of light as the communication speed, the synchronization of the globe time can be established by radio waves. After synchronization, the spatial position at any time can be expressed by $P(\boldsymbol{r}, t)$, where $\boldsymbol{r}$ and $t$ are mutually independent parameters.

The communication speed $c$ used for synchronization is simply called signal speed as it has unique significance in our theory. Signal speed is a system constant, not a universal constant. Both the speed of light and the speed of sound can be used as signal speed.

\subsubsection{Particle and movement}

If particle $P_{i}$ has mass $M_{i}$, and its center of mass is at position $\boldsymbol{r}_{i}=\overrightarrow{O P}_{l}=\left(x_{i}, y_{i}, z_{i}\right)$, let $\boldsymbol{r}_{i}(t)$ be the particle position at time $t$, the movement of particle can be expressed by a set of discontinuous positions $\mathrm{r}_{i}(\tilde{t})$ with the timon $t_{s}$

$$
\mathbb{r}_{i}(\tilde{t})=\left\langle\boldsymbol{r}_{i}(0), \boldsymbol{r}_{i}(1), \cdots, \boldsymbol{r}_{i}(k), \cdots, \boldsymbol{r}_{i}\left(N_{t}\right)\right\rangle .
$$

By the convention of set theory, unordered elements are put in the braces \{\} , and ordered elements are put in the brackets \langle\rangle .

In fact, $\mathbb{r}_{i}(\tilde{t})$ is the sample set of $\mathbb{r}_{i}(t)$ with the sampling interval $t_{s}$ and the sampling number $N_{t}+1$. The position set of the particle system is $\left\{\mathrm{r}_{i}(\tilde{t})\right\}$ with the sampling number $N\left(N_{t}+1\right)$. Our theory uses discrete sets of positions to describe the motion of particles. This description is not only different from the continuous trajectory in classical mechanics, but also different from the wave function in quantum mechanics.

\subsubsection{Displacement and velocity}

If the particle $P_{i}$ moves along a curve $C_{i}$, as shown in Fig. 1, the displacement of $P_{i}$ at any time $\tilde{t}=k$ is defined by

$$
\Delta \boldsymbol{r}_{i}(k) \equiv \boldsymbol{r}_{i}(k+1)-\boldsymbol{r}_{i}(k)=r_{s} \cdot \Delta \tilde{\boldsymbol{r}}_{i}(k) ; \quad \Delta \tilde{\boldsymbol{r}}_{i}(k)=\tilde{\boldsymbol{r}}_{i}(k+1)-\tilde{\boldsymbol{r}}_{i}(k) .
$$


The displacement is the distance between the centers of mass before and after the movement, rather than the arc-length along the moving trajectory. The displacement is independent of the origin point because $\Delta \boldsymbol{r}_{i}(k)=\overrightarrow{O P_{l}(k+1)}-\overrightarrow{O P_{l}(k)}=\overrightarrow{P_{l}(k) P_{l}(k+1)}$.

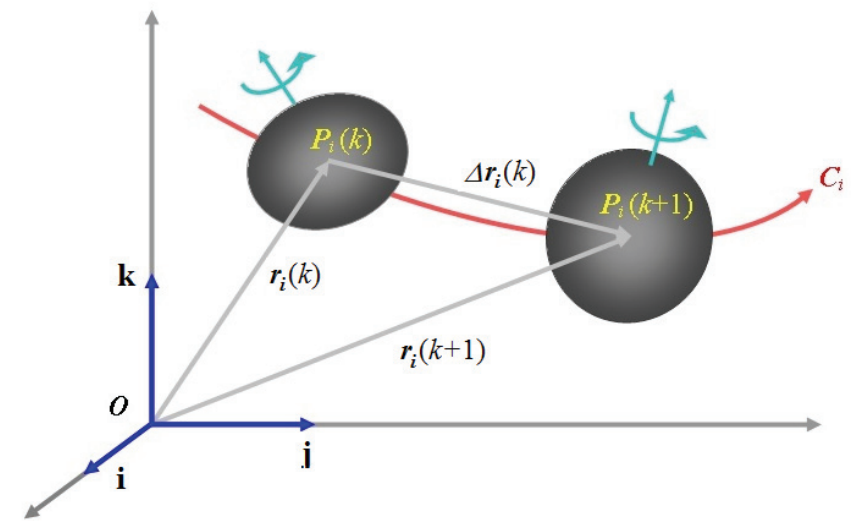

Figure 1. Cartesian coordinate system, position vector of particle, and displacement.

The velocity at any time is defined as the displacement divided by timon,

$$
\boldsymbol{u}_{i}(k) \equiv \frac{\Delta \boldsymbol{r}_{i}(k)}{t_{s}}=\frac{r_{s}}{t_{s}} \cdot \Delta \tilde{\boldsymbol{r}}_{i}(k)=u_{s} \cdot \widetilde{\boldsymbol{u}}_{i}(k) ; \quad u_{s}=\frac{r_{s}}{t_{s}}, \quad \widetilde{\boldsymbol{u}}_{i}(k)=\Delta \tilde{\boldsymbol{r}}_{i}(k) .
$$

In above formula, $\boldsymbol{u}_{s}$ is the scale of velocity, $\widetilde{\boldsymbol{u}}_{i}(k)$ is the digit of velocity. The set of a particle velocity is

$$
\mathbb{U n}_{i}(\tilde{t})=\left\langle\boldsymbol{u}_{i}(0), \boldsymbol{u}_{i}(1), \cdots, \boldsymbol{u}_{i}(k), \cdots, \boldsymbol{u}_{i}\left(N_{t}-1\right)\right\rangle \text {, }
$$

with sampling number $N_{t}$. The set of velocity of the particle system is $\left\{\mathbb{u}_{i}(\tilde{t})\right\}$ with sampling number $N \cdot N_{t}$. The velocity is independent of the reference point of space and time, such property is called the origin irrelevance.

\subsubsection{Volume repulsion}

The spacing between two particles at any time $\boldsymbol{s}_{i j}(k)$ is origin irrelevance, because

The length of spacing is expressed by

$$
\boldsymbol{s}_{i j}(k) \equiv \boldsymbol{r}_{j}(k)-\boldsymbol{r}_{i}(k)=\overrightarrow{O P_{J}}-\overrightarrow{O P_{l}}=\overrightarrow{P_{l} P_{J}}, \quad i \neq j .
$$

$$
s_{i j} \equiv\left|\overrightarrow{P_{l}} P_{J}\right|=\sqrt{\boldsymbol{s}_{i j} \cdot \boldsymbol{s}_{i j}}>0,
$$

where $i \neq j$ or $s_{i j}>0$ is a mathematical expression for the theorem of volume repulsion.

The set of spacing of all particles at any time $k$ is

$$
\mathbb{s}(k)=\left\{\boldsymbol{s}_{i j}(k) \mid i, j=1,2, \cdots, N ; i \neq j\right\}
$$

with sampling number $N(N-1)$. The set of spacing of all particles at all times is

$$
\langle\mathbb{s}(\tilde{t})\rangle=\left\langle\mathbb{s}(0), \mathbb{s}(1), \cdots, \mathbb{s}(k), \cdots, \mathbb{s}\left(N_{t}\right)\right\rangle
$$

with sampling number $N(N-1)\left(N_{t}+1\right)$. The set of spacing $\langle\mathbb{s}(\tilde{t})\rangle$ is a discrete description of the configuration of a particle system.

\subsection{Principle of Objectivity}

\subsubsection{Concepts and axioms} $\tilde{\boldsymbol{x}}$

(a) Physical quantity. Any physical quantity $\boldsymbol{x}$ can be decomposed as the product of scale $x_{\boldsymbol{s}}$ and digit

$$
\boldsymbol{x}=x_{s} \cdot \tilde{\boldsymbol{x}}=x_{s} \times \tilde{\boldsymbol{x}} ; \quad x_{s}>0 .
$$

The scale is the unit of measurement and the identification of the physical quantity, while the digit is the number of the physical quantity.

The scale is a scalar in the domain of positive real number. Depending on the type of physical quantity, the digit can be a scalar, a vector or a tensor. For examples, the digit of time $(\tilde{t})$ is scalar, and the digits of position and velocity $(\tilde{\boldsymbol{r}}, \tilde{\boldsymbol{u}})$ are vectors.

(b) Objective and subjective quantity. The physical quantity $\boldsymbol{x}$ is objective quantity, and the scale $x_{\boldsymbol{s}}$ is 
subjective quantity.

(c) Absolute and relative quantity. The physical quantity $\boldsymbol{x}$ is absolute quantity, and the digit $\widetilde{\boldsymbol{x}}$ is relative quantity.

(d) Axiom of objectivity (Axiom 4). The physical quantities are objective and the laws of motion are objective. Physical theory must exclude all subjective factors.

The objectivity axiom requires the object measurability, the origin irrelevance, and the scale irrelevance.

(e) Object measurability. Object is measurable, unmeasurable things are subjective. A measurable quantity is finite and has scale, which requires $x_{s}>0$. Object measurability excludes the infinity $( \pm \infty)$ from physics.

(f) Origin irrelevance. The reference points of space and time are subjective and relative. All physical formulas must be independent of the origins of space and time.

(g) Scale irrelevance. Any physical relation $z=R(x, y)$ is independent of the scales, i.e. the physical relation and digital relation are equivalent,

$$
z=R(x, y)=z_{S} \cdot \tilde{z} ; \quad \tilde{z}=R(\tilde{x}, \tilde{y}) .
$$

Real objects are objective and measurable. Physical quantities must contain scales. The scale expression (22) is the basis of discretization and digitization of physical quantity. The scale is the quantitative standard and essentially is the quantum. The axiom of objectivity is essentially the principle of universality. The origin irrelevance means that the physical laws are applicable to any place and any time. The scale irrelevance means that the physical laws are applicable to any scale. For example, there should be no difference between macrophysics and microphysics.

\subsubsection{Calculation rules}

The scale irrelevance requires the physical quantities satisfy following algorithms.

(a) Addition and subtraction.

$$
\begin{gathered}
z=x \pm y=x_{s} \cdot \tilde{x} \pm y_{s} \cdot \tilde{y}=x_{s} \cdot(\tilde{x} \pm \tilde{y})=z_{s} \cdot \tilde{z} \\
z_{s}=x_{s}=y_{s}, \quad \tilde{z}=\tilde{x} \pm \tilde{y} .
\end{gathered}
$$

(b) Multiplication.

$$
\begin{gathered}
z=x \cdot y=\left(x_{s} \cdot \tilde{x}\right) \cdot\left(y_{s} \cdot \tilde{y}\right)=\left(x_{s} \cdot y_{s}\right) \cdot(\tilde{x} \cdot \tilde{y})=z_{s} \cdot \tilde{z} \\
z_{s}=x_{s} \cdot y_{s}, \quad \tilde{z}=\tilde{x} \cdot \tilde{y} .
\end{gathered}
$$

(c) Division.

$$
z=\frac{y}{x}=\frac{y_{s} \cdot \tilde{y}}{x_{s} \cdot \tilde{x}}=\frac{y_{s}}{x_{s}} \cdot \frac{\tilde{y}}{\tilde{x}}=z_{s} \cdot \tilde{z} ; \quad z_{s}=\frac{y_{s}}{x_{s}}, \quad \tilde{z}=\frac{\tilde{y}}{\tilde{x}} .
$$

(d) Difference.

$$
\Delta x_{k}=x_{k+1}-x_{k}=x_{s} \cdot\left(\tilde{x}_{k+1}-\tilde{x}_{k}\right)=x_{s} \cdot \Delta \tilde{x}_{k} ; \quad \Delta \tilde{x}_{k}=\tilde{x}_{k+1}-\tilde{x}_{k} .
$$

(e) Integral.

$$
\begin{gathered}
z(n)=\sum_{k=1}^{n}\left[y\left(x_{k}\right) \cdot \Delta x_{k}\right]_{\Delta x_{k}=x_{s}}=\left(y_{s} \cdot x_{s}\right) \cdot \sum_{k=1}^{n} \tilde{y}\left(x_{k}\right)=z_{s} \cdot \tilde{z} ; \\
z_{s}=y_{s} \cdot x_{s}, \quad \tilde{z}=\sum_{k=1}^{n} \tilde{y}\left(x_{k}\right) .
\end{gathered}
$$

(f) Difference quotient.

$$
\frac{d y}{d x}=\left(\frac{\Delta y}{\Delta x}\right)_{\Delta \tilde{x}=1}=\frac{y_{s}}{x_{s}} \cdot\left(\frac{\Delta \tilde{y}}{\Delta \tilde{x}}\right)_{\Delta \tilde{x}=1}=\frac{y_{s}}{x_{s}} \cdot \Delta \tilde{y} .
$$

(g) Differential quotient.

$$
\frac{\tilde{d} y}{\tilde{d} x}=\left(\frac{\Delta y}{\Delta x}\right)_{\Delta \tilde{x} \rightarrow 0}=\frac{y_{s}}{x_{s}} \cdot\left(\frac{\Delta \tilde{y}}{\Delta \tilde{x}}\right)_{\Delta \tilde{x} \rightarrow 0}=\frac{y_{s}}{x_{s}} \cdot \frac{d \tilde{y}}{d \tilde{x}} .
$$

(h) Others. It is required that $x_{s}=1$ for the exponential, logarithmic and trigonometric operations.

$$
e^{x}=e^{x_{s} \cdot \tilde{x}}=\left(e^{x_{s}}\right)^{\tilde{x}}=e^{\tilde{x}},
$$

\subsubsection{Basic physical quantity}

$$
\begin{gathered}
\ln x=\ln \left(x_{s} \cdot \tilde{x}\right)=\ln x_{s}+\ln \tilde{x}=\ln \tilde{x}, \\
\sin x=\sin \left(x_{s} \cdot \tilde{x}\right)=\sin \tilde{x} .
\end{gathered}
$$

Table 1 lists the basic physical quantities and their scale relations. If the signal speed $c$ is used as the velocity scale, i.e. $u_{s}=c$, we get the relation between spacon and timon $r_{s}=c t_{s}$. This relationship is the base for the discretization of space and time. In addition, the scale of kinetic energy becomes $K_{s}=M_{s} c^{2}$. It 
reveals that the Einstein's mass-energy equation is a scale relation in nature.

Table 1. Basic physical quantities and scale relations

\begin{tabular}{|c|c|c|}
\hline Name & Definition & Scale \\
\hline Position & $\boldsymbol{r}=r_{s} \cdot \tilde{\boldsymbol{r}}$ & $r_{s}=u_{s} t_{s}=c t_{s}$ \\
\hline Time & $t=t_{s} \cdot \tilde{t} ; \quad \tilde{t}=0,1,2, \cdots, k, \cdots, N_{t}$ & $t_{s}$ \\
\hline Mass & $M=M_{S} \cdot \widetilde{M}$ & $M_{S}$ \\
\hline Volume & $V=V_{S} \cdot \tilde{V}$ & $V_{s}=r_{s}^{3}$ \\
\hline Particle number & $N=N_{S} \cdot \widetilde{N} ; \quad \widetilde{N}=1,2,3, \cdots$ & $N_{S}=1$ \\
\hline Particle density & $n=N / V=n_{s} \cdot \tilde{n}$ & $n_{s}=1 / V_{s}$ \\
\hline Mass density & $\rho=M / V=\rho_{s} \cdot \tilde{\rho}$ & $\rho_{s}=M_{s} / V_{s}$ \\
\hline Velocity & $\boldsymbol{u}=\left(\frac{\Delta \boldsymbol{r}}{\Delta t}\right)_{\Delta \tilde{t}=1}=u_{S} \cdot \widetilde{\boldsymbol{u}}$ & $u_{s}=\frac{r_{s}}{t_{s}}=c$ \\
\hline Momentum & $\boldsymbol{p}=M \boldsymbol{u}=p_{s} \cdot \widetilde{\boldsymbol{p}}$ & $p_{s}=M_{s} u_{s}$ \\
\hline Kinetic energy & $K=(1 / 2) \boldsymbol{p} \cdot \boldsymbol{u}=K_{S} \cdot \widetilde{K}$ & $K_{s}=p_{s} u_{s}=M_{s} u_{s}^{2}$ \\
\hline
\end{tabular}

\section{$3 \quad$ Theory of Particle Field}

\subsection{Density Field}

\subsubsection{Grid and gridon}

Let's choose the spacon $r_{s}$ and determine the scale of volume $V_{s}=r_{s}^{3}$ which is termed volumon. Then, we use $V_{s}$ to divide the object into grids in the number $N_{q}=V / V_{s}$ without gaps. Each grid is a cube denoted by $G_{q}$. All grids form a lattice denoted by the grid set $\mathbb{G}$,

$$
\mathbb{G}=\left\{G_{q} \mid q=1,2, \cdots, N_{q}\right\} .
$$

After that, we can use the signal speed $c$ to determine the timon $t_{s}=r_{s} / c$, and divide the time into discrete series. Denoted the grid position by vector $\boldsymbol{r}_{q}$, the grid $G_{q}$ at any time is expressed by $G\left(\boldsymbol{r}_{q}, \tilde{t}\right)$. For convenience, Fig. 2(a) gives a schematic diagram of two-dimensional lattice of square grid as the example.

We can understand the grids from another perspective. If we regard the particles in a grid as a transient particle $\mathbb{G}_{q}$, termed gridon, then the object is a set of gridons

$$
\text { Objீẹt }=\left\{\mathbb{G}_{q} \mid q=1,2, \cdots, N_{q}\right\} .
$$

Each gridon has volume $V_{s}$ and mass $M_{q}$. The number of gridons $N_{q}$ can vary with the volume $V$ while keep the volumon $V_{s}$ constant. Different from the concept of fluid parcel [12], a gridon has variable mass and invariant shape (cube).



(a)

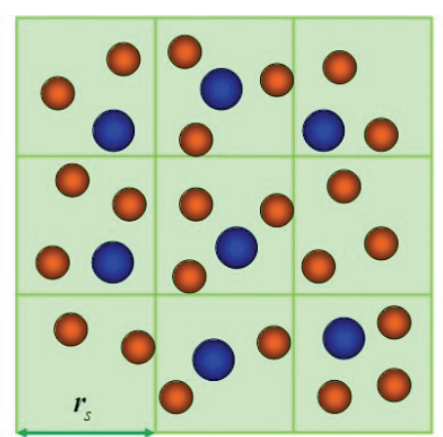

(b)

Figure 2. (a) Schematic diagram of two-dimensional lattice of square grids. (b) An example of configuration of particles for the calculation of mass density and mass potential. 


\subsubsection{Mass density}

The density of an object can be calculated based on the grids. A grid $G_{q}$ contains $n_{q}$ particles. If the mass of the $i$-particle is $M_{i q}$, the mass in grid is the sum $M_{q}=\sum_{i=1}^{n_{q}} M_{i q}$. Then, we can determine the mass density $\rho_{q}$,

$$
\rho_{q}=\frac{M_{q}}{V_{s}}=\frac{M_{s}}{V_{s}} \cdot\left(\sum_{i=1}^{n_{q}} \tilde{M}_{i q}\right)=\rho_{s} \cdot \tilde{\rho}_{q} ; \quad \rho_{s}=\frac{M_{s}}{V_{s}}, \quad \tilde{\rho}_{q}=\sum_{i=1}^{n_{q}} \tilde{M}_{i q} .
$$

The total number of particles and the total mass of object are $N=\sum_{q=1}^{N_{q}} n_{q}$ and $M=\sum_{q=1}^{N_{q}} M_{q}$, respectively. The average density of gridons is $\bar{n}=N / N_{q}$.

For example, we consider an object containing 9 grids and 30 particles as shown in Fig. 2(b). All particles do not intersect each other according to the theorem of volume repulsion. Suppose the particles of the first type have 23, each of which has the mass $M_{1}=M_{s}$. The particles of the second type have 7 , each of which has the mass $M_{2}=2 M_{s}$. Table 2 gives the digits of mass density calculated from the particle configuration of Fig. 2(b).

Table 2. Mass density and mass potential based on the configuration of particles in Fig. 2(b).

\begin{tabular}{cccccccccc}
\hline$q$ & 1 & 2 & 3 & 4 & 5 & 6 & 7 & 8 & 9 \\
\hline$\tilde{\rho}_{q}$ & 2 & 4 & 5 & 5 & 5 & 3 & 4 & 5 & 4 \\
\hline$-4 \pi \widetilde{\Phi}_{q}$ & 22.0 & 23.7 & 20.4 & 22.9 & 27.6 & 25.6 & 21.4 & 23.8 & 20.8 \\
\hline
\end{tabular}

\subsubsection{Momentum density}

If the momentum of the $i$-particle in the grid $G_{q}$ is $\boldsymbol{p}_{i q}=M_{i q} \boldsymbol{u}_{i q}$, the sum of momentum in $G_{q}$ is $\boldsymbol{p}_{q}=\sum_{i=1}^{n_{q}} \boldsymbol{p}_{i q}$. Then, we get the momentum density $\boldsymbol{j}_{q}$,

$$
\boldsymbol{j}_{q}=\frac{\boldsymbol{p}_{q}}{V_{s}}=\frac{p_{s}}{V_{s}} \cdot\left(\sum_{i=1}^{n_{q}} \tilde{\boldsymbol{p}}_{i q}\right)=j_{s} \cdot \tilde{\boldsymbol{\jmath}}_{q} ; \quad j_{s}=\frac{p_{s}}{V_{s}}, \quad \tilde{\boldsymbol{\jmath}}_{q}=\sum_{i=1}^{n_{q}} \tilde{\boldsymbol{p}}_{i q} .
$$

The total momentum of an object is the sum $\boldsymbol{p}=\sum_{q=1}^{N_{q}} \boldsymbol{p}_{q}$. The distributions of the mass density $\rho_{q}$ and the momentum density $\boldsymbol{j}_{q}$ are referred to as density field. It can be imagined as a set of gridons with volume $V_{s}$, mass $M_{q}$ and momentum $\boldsymbol{p}_{q}$.

A velocity field $\boldsymbol{u}_{q}$ can be calculated by the momentum density $\boldsymbol{j}_{q}$ and the mass density $\rho_{q}$,

$$
\boldsymbol{u}_{q}=\frac{\boldsymbol{j}_{q}}{\rho_{q}}=\frac{p_{s}}{M_{s}} \cdot \frac{\sum_{i=1}^{n_{q}} \widetilde{\boldsymbol{p}}_{i q}}{\sum_{i=1}^{n_{q}} \widetilde{M}_{i q}}=u_{s} \cdot \widetilde{\boldsymbol{u}}_{q} ; \quad u_{s}=\frac{p_{s}}{M_{s}}, \widetilde{\boldsymbol{u}}_{q}=\frac{\sum_{i=1}^{n_{q}} \widetilde{\boldsymbol{p}}_{i q}}{\sum_{i=1}^{n_{q}} \widetilde{M}_{i q}},
$$

where $\boldsymbol{u}_{q}$ can be thought as the velocity of the gridon $\mathbb{G}_{q}$.

\section{$3.2 \quad$ Potential Field}

\subsubsection{Mass potential}

Mass potential $\Phi_{q}$ is constructed by the gridon mass and the gridon spacing,

$$
\Phi_{q}\left(\boldsymbol{x}_{q}\right)=-\frac{1}{\varphi} \sum_{k=1, k \neq q}^{N_{q}} \frac{M_{k}^{\prime}}{\left|\boldsymbol{x}_{q}-\boldsymbol{x}_{k}^{\prime}\right|}=-\frac{1}{\varphi} \sum_{k=1, k \neq q}^{N_{q}} \frac{M_{k}^{\prime}}{r_{k q}},
$$

where $\boldsymbol{x}_{q}$ is the position of field grid $G_{q}$, and $\boldsymbol{x}_{k}^{\prime}$ is the position of source grid $G_{k}^{\prime} \cdot r_{k q}=\left|\boldsymbol{x}_{q}-\boldsymbol{x}_{k}^{\prime}\right|$ is the spacing between $G_{k}^{\prime}$ and $G_{q} . k \neq q$ excludes the case of $r_{k q}=0$. The medium constant $\varphi$ is defined by

$$
\varphi=\tilde{\varphi} \cdot \varphi_{s} \equiv 4 \pi \varphi_{s} ; \quad \tilde{\varphi} \equiv 4 \pi .
$$

The mass potential $\Phi_{q}$ includes the mass contribution from all gridons except for $M_{q}$. Now, we define the potential energy of the gridon $\mathbb{G}_{q}$ as $J_{q} \equiv M_{q} \Phi_{q}$, the total potential energy $J$ can be obtained by 


$$
J=\sum_{q=1}^{N_{q}} J_{q} \equiv \sum_{q=1}^{N_{q}} M_{q} \Phi_{q}=-\frac{1}{\varphi} \sum_{q=1}^{N_{q}}\left(\sum_{k=1, k \neq q}^{N_{q}} \frac{M_{q} M_{k}^{\prime}}{r_{k q}}\right) .
$$

Since the scale of energy is $J_{s}=M_{s} u_{s}^{2}$, the scales of $\Phi$ and $\varphi$ can be determined as

$$
\Phi_{s}=\frac{J_{s}}{M_{s}}=u_{s}^{2} ; \quad \varphi_{s}=\frac{M_{s}}{r_{s} \Phi_{s}}=\frac{M_{s}}{r_{s} u_{s}^{2}} .
$$

The scale $\Phi_{s}=u_{s}^{2}$ indicates that the mass potential stands for the square of velocity. The digits of mass potential calculated from the particle configuration of Fig. 2(b) are listed in Table 2.

\subsubsection{Momentum potential}

Momentum potential $\boldsymbol{A}_{q}$ is constructed by the gridon momentum and the gridon spacing,

$$
\boldsymbol{A}_{q}\left(\boldsymbol{x}_{q}\right)=\alpha \cdot \sum_{k=1, k \neq q}^{N_{q}} \frac{\boldsymbol{p}_{k}^{\prime}}{\left|\boldsymbol{x}_{q}-\boldsymbol{x}_{k}^{\prime}\right|}=\alpha \cdot \sum_{k=1, k \neq q}^{N_{q}} \frac{\boldsymbol{p}_{k}^{\prime}}{r_{k q}},
$$

where $\alpha$ is the dynamic constant

$$
\alpha=\tilde{\alpha} \cdot \alpha_{s} \equiv(4 \pi)^{-1} \alpha_{s} ; \quad \tilde{\alpha} \equiv(4 \pi)^{-1} .
$$

The momentum potential $\boldsymbol{A}_{q}$ includes the momentum contribution from all gridons except for $\boldsymbol{p}_{q}$. Obviously, the direction of $\boldsymbol{A}_{q}$ is not consistent with the direction of $\boldsymbol{p}_{q}$. Now, we define the kinetic energy of the gridon $\mathbb{G}_{q}$ as $K_{q} \equiv \boldsymbol{p}_{q} \cdot \boldsymbol{A}_{q}$, the total kinetic energy $K$ can be obtained by

$$
K=\sum_{q=1}^{N_{q}} K_{q} \equiv \sum_{q=1}^{N_{q}}\left(\boldsymbol{p}_{q} \cdot \boldsymbol{A}_{q}\right)=\alpha \sum_{q=1}^{N_{q}}\left(\sum_{k=1, k \neq q}^{N_{q}} \frac{\boldsymbol{p}_{q} \cdot \boldsymbol{p}_{k}^{\prime}}{r_{k q}}\right) .
$$

Since the scale of energy is $K_{s}=M_{s} u_{s}^{2}$, the scales of $\boldsymbol{A}$ and $\alpha$ can be determined as

$$
A_{s}=\frac{K_{s}}{p_{s}}=u_{s} ; \quad \alpha_{s}=\frac{A_{s} r_{s}}{p_{s}}=\frac{r_{s}}{M_{s}} .
$$

The scale $A_{s}=u_{s}$ indicates that the momentum potential stands for the velocity. According to Eqs. (39) and (43), we get the relation between the medium constant and the dynamic constant

$$
\alpha \varphi=\alpha_{s} \varphi_{s}=1 / u_{s}^{2} .
$$

The distributions of mass potential and momentum potential are referred to as potential field. In fact, the potential field is the spatially correlative function of the density field, which decays with the spatial distance.

\subsection{Continuity and Conservation}

\subsubsection{Continuous field}

If $N_{q} \ll N$, then $\bar{n}=N / N_{q} \gg 1$, the average particle density is enormous. In such case, the mass density, momentum density and velocity can be expressed by continuous functions

$$
\rho=\rho(\boldsymbol{x}, t), \quad \boldsymbol{j}=\boldsymbol{j}(\boldsymbol{x}, t), \quad \boldsymbol{u}=\boldsymbol{u}(\boldsymbol{x}, t) .
$$

The total mass $M(t)$ and the total momentum $\boldsymbol{p}(t)$ of an object are expressed in integral form

$$
M(t)=\iiint_{V} \rho\left(\boldsymbol{x}^{\prime}, t\right) d \boldsymbol{x}^{\prime} ; \quad \boldsymbol{p}(t)=\iiint_{V} \boldsymbol{j}\left(\boldsymbol{x}^{\prime}, t\right) d \boldsymbol{x}^{\prime} .
$$

Consequently, we have the continuous forms of the mass potential and the momentum potential

$$
\begin{gathered}
\Phi(\boldsymbol{x}, t)=\frac{-1}{\varphi} \iiint_{V} \frac{\rho\left(\boldsymbol{x}^{\prime}, t\right)}{\left|\boldsymbol{x}-\boldsymbol{x}^{\prime}\right|} d \boldsymbol{x}^{\prime}=\frac{-1}{4 \pi \varphi_{s}} \iiint_{V} \frac{\rho\left(\boldsymbol{x}^{\prime}, t\right)}{r} d \boldsymbol{x}^{\prime}, \\
\boldsymbol{A}(\boldsymbol{x}, t)=\alpha \iiint_{V} \frac{\boldsymbol{j}\left(\boldsymbol{x}^{\prime}, t\right)}{\left|\boldsymbol{x}-\boldsymbol{x}^{\prime}\right|} d \boldsymbol{x}^{\prime}=\frac{\alpha_{s}}{4 \pi} \iiint_{V} \frac{\boldsymbol{j}\left(\boldsymbol{x}^{\prime}, t\right)}{r} d \boldsymbol{x}^{\prime}, \\
r=\left|\boldsymbol{x}-\boldsymbol{x}^{\prime}\right|=\sqrt{\left(x_{1}-x_{1}^{\prime}\right)^{2}+\left(x_{2}-x_{2}^{\prime}\right)^{2}+\left(x_{3}-x_{3}^{\prime}\right)^{2}} .
\end{gathered}
$$

The total potential energy and the total kinetic energy become

$$
J(t)=\iiint_{V} \rho\left(\boldsymbol{x}^{\prime}, t\right) \Phi\left(\boldsymbol{x}^{\prime}, t\right) d \boldsymbol{x}^{\prime}, \quad K(t)=\iiint_{V} \boldsymbol{j}\left(\boldsymbol{x}^{\prime}, t\right) \cdot \boldsymbol{A}\left(\boldsymbol{x}^{\prime}, t\right) d \boldsymbol{x}^{\prime} .
$$

The spatial reference system in the case of continuous field is shown in Fig. 3, where $V$ is the volume of object, $S$ is the surface of boundary, and $\boldsymbol{n}$ is the outward-pointing unit normal vector. The volume $V$ and boundary $S$ are both changeable with time. In the case of continuity, a set of particle field equations 
can be derived by vector calculus. The identities of vector calculus can be found in relevant textbooks, unless necessary, they will not be specified in this article.

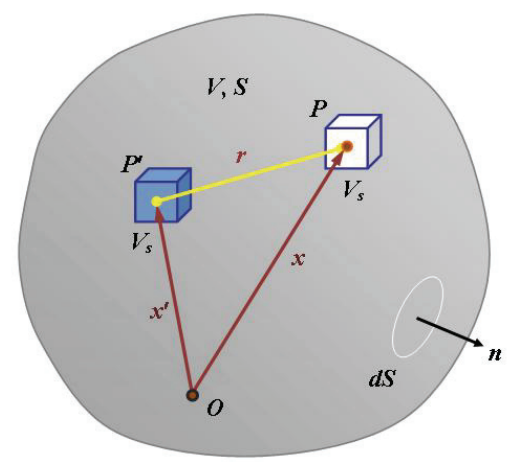

Figure 3. Continuous field and reference system

\subsubsection{Continuity formula}

If $\boldsymbol{y}(\boldsymbol{x}, t)$ is the bulk density of any physical quantity $\boldsymbol{Y}(t)$, then

$$
\boldsymbol{Y}(t)=\iiint_{V} \boldsymbol{y}\left(\boldsymbol{x}^{\prime}, t\right) d \boldsymbol{x}^{\prime} .
$$

The time derivative of $\boldsymbol{Y}(t)$ can be expressed as

$$
\frac{d \boldsymbol{Y}(t)}{d t}=\frac{d}{d t} \iiint_{V} \boldsymbol{y}\left(\boldsymbol{x}^{\prime}, t\right) d \boldsymbol{x}^{\prime} \equiv \iiint_{V} \frac{D \boldsymbol{y}}{D t} d \boldsymbol{x}^{\prime} .
$$

where $D \boldsymbol{y} / D t$ is called total derivative of $\boldsymbol{y}$. As density $\boldsymbol{y}$ and volume $V$ are both changeable, we have

where

$$
\begin{aligned}
\frac{d \boldsymbol{Y}}{d t} & \equiv \iiint_{V} \frac{D \boldsymbol{y}}{D t} d \boldsymbol{x}^{\prime}=\frac{1}{t_{s}}\left[\iiint_{V+\Delta V} \boldsymbol{y}\left(\boldsymbol{x}^{\prime}, t+t_{s}\right) d \boldsymbol{x}^{\prime}-\iiint_{V} \boldsymbol{y}\left(\boldsymbol{x}^{\prime}, t\right) d \boldsymbol{x}^{\prime}\right] \\
& =\frac{1}{t_{s}}\left[\iiint_{V} \boldsymbol{y}\left(\boldsymbol{x}^{\prime}, t+t_{s}\right) d \boldsymbol{x}^{\prime}-\iiint_{V} \boldsymbol{y}\left(\boldsymbol{x}^{\prime}, t\right) d \boldsymbol{x}^{\prime}\right]+\frac{1}{t_{s}} \iiint_{\Delta V} \boldsymbol{y}\left(\boldsymbol{x}^{\prime}, t\right) d \boldsymbol{x}^{\prime} \\
& =\iiint_{V} \frac{\partial \boldsymbol{y}}{\partial t} d \boldsymbol{x}^{\prime}+I(\Delta V),
\end{aligned}
$$

$$
\begin{gathered}
\iiint_{V} \frac{\partial \boldsymbol{y}}{\partial t} d \boldsymbol{x}^{\prime}=\frac{1}{t_{s}}\left[\iiint_{V} \boldsymbol{y}\left(\boldsymbol{x}^{\prime}, t+t_{s}\right) d \boldsymbol{x}^{\prime}-\iiint_{V} \boldsymbol{y}\left(\boldsymbol{x}^{\prime}, t\right) d \boldsymbol{x}^{\prime}\right], \\
I(\Delta V)=\frac{1}{t_{s}} \iiint_{\Delta V} \boldsymbol{y}\left(\boldsymbol{x}^{\prime}, t\right) d \boldsymbol{x}^{\prime} .
\end{gathered}
$$

Eq. (52a) is a change of $\boldsymbol{Y}$ caused by varying the density $\boldsymbol{y}$ and keeping the volume $V$ invariant. Eq. (52b) is a change of $\boldsymbol{Y}$ caused by varying the volume $V$ and keeping the density $\boldsymbol{y}$ invariant. As the volume element on the boundary can be represented by the area element $d \boldsymbol{x}^{\prime}=\left(\boldsymbol{u} t_{s}\right) \cdot d \boldsymbol{S}^{\prime}, I(\Delta V)$ can be calculated by the surface integral,

$$
\begin{aligned}
I(\Delta V) & =\frac{1}{t_{s}} \iiint_{\Delta V} \boldsymbol{y}\left(\boldsymbol{x}^{\prime}, t\right) d \boldsymbol{x}^{\prime}=\frac{1}{t_{s}} \oiint_{S} \boldsymbol{y}\left(\boldsymbol{u} t_{s}\right) \cdot d \boldsymbol{S}^{\prime} \\
& =\oiint_{S} \boldsymbol{y} \boldsymbol{u} \cdot d \boldsymbol{S}^{\prime}=\iiint_{V} \nabla \cdot(\boldsymbol{y} \boldsymbol{u}) d \boldsymbol{x}^{\prime} .
\end{aligned}
$$

where $\boldsymbol{y} \boldsymbol{u}$ is the dyad of $\boldsymbol{y}$ and $\boldsymbol{u}$. In the last step of above derivation, Gaussian formula of vector integral is applied to convert the closed surface integral back to the volume integral.

Substituting Eq. (53) into Eq. (51), we have

$$
\iiint_{V}\left[\frac{D \boldsymbol{y}}{D t}-\frac{\partial \boldsymbol{y}}{\partial t}-\nabla \cdot(\boldsymbol{y u})\right] d \boldsymbol{x}^{\prime}=0 .
$$

As the volume is arbitrary, we find the expression of total derivative of $\boldsymbol{y}$ as

Eq. (54) is called continuity formula.

$$
\frac{D \boldsymbol{y}}{D t}-\frac{\partial \boldsymbol{y}}{\partial t}-\nabla \cdot(\boldsymbol{y} \boldsymbol{u})=0 \text {. }
$$


Applying Eq. (54b) to mass and momentum density, we get the formulas of mass continuity and momentum continuity, respectively,

\subsubsection{Conservation law}

$$
\begin{aligned}
& \frac{D \rho}{D t}-\frac{\partial \rho}{\partial t}-\nabla \cdot(\rho \boldsymbol{u})=0 \\
& \frac{D \boldsymbol{j}}{D t}-\frac{\partial \boldsymbol{j}}{\partial t}-\nabla \cdot(\boldsymbol{j} \boldsymbol{u})=0 .
\end{aligned}
$$

If the total amount of $\boldsymbol{Y}$ in the volume is time invariant, i.e. $\boldsymbol{Y}(t)=\boldsymbol{Y}$, we have

This is called the law of conservation.

$$
\frac{D \boldsymbol{y}}{D t}=\frac{\partial \boldsymbol{y}}{\partial t}-\nabla \cdot(\boldsymbol{y} \boldsymbol{u})=0 \text {. }
$$

According to the theorem of mass imperishability, the total mass of an object is time invariant, so

$$
\frac{D \rho}{D t}=\frac{\partial \rho}{\partial t}+\nabla \cdot(\rho \boldsymbol{u})=\frac{\partial \rho}{\partial t}+\nabla \cdot \boldsymbol{j}=0 .
$$

Above formula is called the equation of mass conservation.

\subsection{Action Field}

The action field is the space derivative of the potential field. It can be derived with the help of vector calculus [13]. The action field includes gradient field, divergence field, and curl field.

\subsubsection{Gradient field}

The negative gradient of the mass potential is defined as the gradient field $\boldsymbol{G}$,

$$
\begin{gathered}
\boldsymbol{G}(\boldsymbol{x}, t) \equiv-\nabla \Phi=\frac{1}{4 \pi \varphi_{s}} \iiint_{V} \rho\left(\boldsymbol{x}^{\prime}, t\right) \nabla\left(\frac{1}{r}\right) d \boldsymbol{x}^{\prime} \\
=\frac{-1}{4 \pi \varphi_{s}} \iiint_{V} \frac{\rho\left(\boldsymbol{x}^{\prime}, t\right) \boldsymbol{r}}{r^{3}} d \boldsymbol{x}^{\prime} ; \quad G_{s}=\frac{\Phi_{s}}{r_{s}}=\frac{r_{s}}{t_{s}^{2}} .
\end{gathered}
$$

The form of gradient field is similar to the Coulomb law in electrodynamics [13]. The scale $G_{s}=r_{s} / t_{s}^{2}$ indicates that the gradient field stands for acceleration.

The divergence of the gradient field is calculated as follows

$$
\begin{gathered}
\nabla \cdot \boldsymbol{G}=-\nabla^{2} \Phi=\frac{1}{4 \pi \varphi_{s}} \iiint_{V} \rho\left(\boldsymbol{x}^{\prime}, t\right) \nabla^{2}\left(\frac{1}{r}\right) d \boldsymbol{x}^{\prime} \\
=-\frac{1}{\varphi_{s}} \iiint_{V} \rho\left(\boldsymbol{x}^{\prime}, t\right) \delta\left(\boldsymbol{x}-\boldsymbol{x}^{\prime}\right) d \boldsymbol{x}^{\prime}=-\frac{\rho(\boldsymbol{x}, t)}{\varphi_{s}},
\end{gathered}
$$

where $\delta(\boldsymbol{x})$ is Dirac delta. $\nabla \cdot \boldsymbol{G}=-\rho / \varphi_{s}$ is called the Gaussian theorem of gradient field, and $\nabla^{2} \Phi=\rho / \varphi_{s}$ is the Poisson equation of mass potential.

According to vector calculus, the curl of the gradient field is always equal to zero

$$
\nabla \times \boldsymbol{G}=-\nabla \times \nabla \Phi \equiv 0,
$$

which indicates that the gradient field is vortex free.

Take the curl operation on both sides of Eq. (60), we have

therefore

$$
\nabla \times(\nabla \times \boldsymbol{G}) \equiv \nabla(\nabla \cdot \boldsymbol{G})-\nabla^{2} \boldsymbol{G}=-\left(1 / \varphi_{s}\right) \nabla \rho-\nabla^{2} \boldsymbol{G} \equiv 0
$$

$$
\nabla^{2} \boldsymbol{G}=-\nabla \rho / \varphi_{s}
$$

It is the Poisson equation of the gradient field. Since $(4 \pi r)^{-1}$ is the Green function of the open space, there is a general solution to the gradient field if the boundary effect is ignored [14]

$$
\boldsymbol{G}(\boldsymbol{x}, t)=\frac{1}{4 \pi \varphi_{s}} \iiint \frac{\nabla \rho\left(\boldsymbol{x}^{\prime}, t\right)}{r} d \boldsymbol{x}^{\prime} .
$$

It is seen that the gradient field depends on the gradient of the mass density.

\subsubsection{Divergence field}

The divergence of the momentum potential is defined as the divergence field $D$. It is calculated as follows, 


$$
\begin{aligned}
D & \equiv \nabla \cdot \boldsymbol{A}=\frac{\alpha_{s}}{4 \pi} \nabla \cdot\left[\iiint_{V} \frac{\boldsymbol{j}\left(\boldsymbol{x}^{\prime}, t\right)}{r} d \boldsymbol{x}^{\prime}\right] \\
& =\frac{\alpha_{s}}{4 \pi} \iiint_{V} \boldsymbol{j}\left(\boldsymbol{x}^{\prime}, t\right) \cdot \nabla\left(\frac{1}{r}\right) d \boldsymbol{x}^{\prime}=-\frac{\alpha_{s}}{4 \pi} \iiint_{V} \boldsymbol{j}\left(\boldsymbol{x}^{\prime}, t\right) \cdot \nabla^{\prime}\left(\frac{1}{r}\right) d \boldsymbol{x}^{\prime} \\
& =-\frac{\alpha_{s}}{4 \pi} \iiint_{V} \nabla^{\prime} \cdot\left[\frac{\boldsymbol{j}\left(\boldsymbol{x}^{\prime}, t\right)}{r}\right] d \boldsymbol{x}^{\prime}+\frac{\alpha_{s}}{4 \pi} \iiint_{V} \frac{\nabla^{\prime} \cdot \boldsymbol{j}\left(\boldsymbol{x}^{\prime}, t\right)}{r} d \boldsymbol{x}^{\prime} \\
& =-\frac{\alpha_{s}}{4 \pi} \oiint_{S} \frac{\boldsymbol{j}\left(\boldsymbol{x}^{\prime}, t\right) \cdot d \boldsymbol{S}^{\prime}}{r}-\frac{\alpha_{s}}{4 \pi} \iiint_{V} \frac{1}{r} \frac{\partial \rho\left(\boldsymbol{x}^{\prime}, t\right)}{\partial t} d \boldsymbol{x}^{\prime} \\
& =-\frac{\alpha_{s}}{4 \pi} \oiint_{S} \frac{\boldsymbol{j}\left(\boldsymbol{x}^{\prime}, t\right) \cdot d \boldsymbol{S}^{\prime}}{r}-\frac{\alpha_{s}}{4 \pi} \frac{\partial}{\partial t} \iiint_{V} \frac{\rho\left(\boldsymbol{x}^{\prime}, t\right)}{r} d \boldsymbol{x}^{\prime} \\
& =B(S)+\alpha_{s} \varphi_{s} \frac{\partial \Phi}{\partial t}=B(S)+\frac{1}{u_{s}^{2}} \frac{\partial \Phi}{\partial t} .
\end{aligned}
$$

In above derivation, we use the identities of vector calculus and the equation of mass conservation. $B(S)$ stands for the boundary effect. If the boundary effect is ignored, i.e.

$$
B(S)=-\frac{\alpha_{s}}{4 \pi} \oiint_{S} \frac{\boldsymbol{j}\left(\boldsymbol{x}^{\prime}, t\right) \cdot d \boldsymbol{S}^{\prime}}{r}=0,
$$

the divergence field is eventually written as

$$
D \equiv \nabla \cdot \boldsymbol{A}=\frac{1}{u_{s}^{2}} \frac{\partial \Phi}{\partial t} ; \quad D_{s}=\frac{A_{s}}{r_{s}}=\frac{1}{t_{s}} .
$$

The scale $D_{s}=1 / t_{s}$ indicates that the divergence field stands for frequency. Eq. (64) determines the relation between the mass and momentum potentials, and has a similar form to the Lorentz gauge in electrodynamics.

The gradient of the divergence field is calculated as follows

$$
\nabla D=\nabla(\nabla \cdot \boldsymbol{A})=\frac{1}{u_{s}^{2}} \nabla\left(\frac{\partial \Phi}{\partial t}\right)=-\frac{1}{u_{s}^{2}} \frac{\partial \boldsymbol{G}}{\partial t} .
$$

The mutual independence of time and space is taken into consideration in above derivation.

In fact, formula (64) is a wave equation in the form of real number. Let's introduce the wave parameter $\xi$, the circular frequency $\omega$ and the wave vector $\boldsymbol{\kappa}$,

$$
\xi=\boldsymbol{\kappa} \cdot \boldsymbol{x}-\omega t ; \quad \omega=2 \pi v ; \quad \boldsymbol{\kappa}=\nabla \xi ; \kappa=|\boldsymbol{\kappa}|=2 \pi / \lambda,
$$

where $v$ is the frequency and $\lambda$ the wave length. There is a general solution of the wave equation

$$
\Phi_{W}=-W(\xi), \boldsymbol{A}_{W}=(\boldsymbol{\kappa} / \omega) W(\xi) .
$$

$\left(\Phi_{W}, \boldsymbol{A}_{W}\right)$ is called wave potential. It is seen that $\boldsymbol{A}_{W}$ is consistent with the direction of wave vector, representing the direction of wave propagation. For above reasons, we know that the divergence field represents the vibrating frequency.

Substituting the wave potential (67) into the wave equation (64), we have

Eq. (68) gives following relationships,

$$
\nabla \cdot \boldsymbol{A}_{W}=\frac{\kappa^{2}}{\omega} \frac{d W}{d \xi}, \quad \frac{1}{u_{s}^{2}} \frac{\partial \Phi_{W}}{\partial t}=\frac{\omega}{u_{s}^{2}} \frac{d W}{d \xi} .
$$

$$
\omega=\kappa u_{s}, u_{s}=\lambda v=c,
$$

where $c$ is the speed of wave. An important conclusion is that the wave speed should be adopted as the scale of velocity. In such case, we find the scales of length and time as

\subsubsection{Curl field}

$$
r_{s}=\lambda, \quad t_{s}=1 / v \text {. }
$$

The curl of the momentum potential is called curl field $\boldsymbol{C}$,

$$
\begin{gathered}
\boldsymbol{C} \equiv \nabla \times \boldsymbol{A}=\frac{\alpha_{s}}{4 \pi} \iiint_{V} \nabla \times\left[\frac{\boldsymbol{j}\left(\boldsymbol{x}^{\prime}, t\right)}{r}\right] d \boldsymbol{x}^{\prime}=\frac{\alpha_{s}}{4 \pi} \iiint_{V} \nabla\left(\frac{1}{r}\right) \times \boldsymbol{j}\left(\boldsymbol{x}^{\prime}, t\right) d \boldsymbol{x}^{\prime} \\
=-\frac{\alpha_{s}}{4 \pi} \iiint_{V}\left(\frac{\boldsymbol{r}}{r^{3}}\right) \times \boldsymbol{j}\left(\boldsymbol{x}^{\prime}, t\right) d \boldsymbol{x}^{\prime}=\frac{\alpha_{s}}{4 \pi} \iiint_{V} \frac{\boldsymbol{j}\left(\boldsymbol{x}^{\prime}, t\right) \times \boldsymbol{r}}{r^{3}} d \boldsymbol{x}^{\prime} .
\end{gathered}
$$

Omitting derivation process, the curl field is expressed as

$$
\boldsymbol{C} \equiv \nabla \times \boldsymbol{A}=\frac{\alpha_{s}}{4 \pi} \iiint_{V} \frac{\boldsymbol{j}\left(\boldsymbol{x}^{\prime}, t\right) \times \boldsymbol{r}}{r^{3}} d \boldsymbol{x}^{\prime} ; \quad C_{s}=\frac{A_{s}}{r_{s}}=\frac{1}{t_{s}} .
$$


The form of curl field is similar to the Biot-Savart law in electrodynamics. Because of the scale $C_{s}=1 / t_{s}$, the curl field stands for the spin frequency.

The divergence of the curl field is always equal to zero because $\boldsymbol{C}$ is the curl of $\boldsymbol{A}$

$$
\nabla \cdot \boldsymbol{C}=\nabla \cdot(\nabla \times \boldsymbol{A}) \equiv 0 .
$$

Laplace operator acts on the momentum potential gives the Poisson equation of the momentum potential

$$
\nabla^{2} \boldsymbol{A}=\frac{\alpha_{s}}{4 \pi} \iiint_{V} \boldsymbol{j}\left(\boldsymbol{x}^{\prime}, t\right) \nabla^{2}\left(\frac{1}{r}\right) d \boldsymbol{x}^{\prime}=-\alpha_{s} \iiint_{V} \boldsymbol{j}\left(\boldsymbol{x}^{\prime}, t\right) \delta\left(\boldsymbol{x}-\boldsymbol{x}^{\prime}\right) d \boldsymbol{x}^{\prime}=-\alpha_{s} \boldsymbol{j}(\boldsymbol{x}, t) .
$$

The curl of the curl field can be obtained by quoting above result as

$$
\nabla \times \boldsymbol{C}=\nabla \times(\nabla \times \boldsymbol{A}) \equiv \nabla(\nabla \cdot \boldsymbol{A})-\nabla^{2} \boldsymbol{A}=\nabla D+\alpha_{s} \boldsymbol{j} .
$$

Substituting Eq. (65) into Eq. (74), we find

$$
\nabla \times \boldsymbol{C}=\alpha_{s} \boldsymbol{j}-\frac{1}{u_{s}^{2}} \frac{\partial \boldsymbol{G}}{\partial t} .
$$

Applying the curl operator on the leftmost side of Eq. (74) and quoting the identity $\nabla \cdot \boldsymbol{C} \equiv 0$, it has

$$
\nabla \times(\nabla \times \boldsymbol{C}) \equiv \nabla(\nabla \cdot \boldsymbol{C})-\nabla^{2} \boldsymbol{C}=-\nabla^{2} \boldsymbol{C} .
$$

Applying the curl operator on the rightmost side of Eq. (74) and quoting the identity $\nabla \times \nabla D \equiv 0$, it has

$$
\nabla \times\left(\nabla D+\alpha_{s} \boldsymbol{j}\right)=\alpha_{s} \nabla \times \boldsymbol{j}
$$

Since Eq. (76) and Eq. (77) are equal, we finally obtain

$$
\nabla^{2} \boldsymbol{C}=-\alpha_{s} \nabla \times \boldsymbol{j}
$$

Eq. (78) is the Poisson equation of the curl field. There is a general solution to the curl field if the boundary effect is ignored

$$
\boldsymbol{C}(\boldsymbol{x}, t)=\frac{\alpha_{s}}{4 \pi} \iiint_{V} \frac{\nabla \times \boldsymbol{j}\left(\boldsymbol{x}^{\prime}, t\right)}{r} d \boldsymbol{x}^{\prime} .
$$

It is seen that the curl field depends on the curl of the momentum density.

\subsection{Equations of Particle Field}

The equations of particle field are derived from mass density and momentum density. The density field is the exact solution to the equations. In principle, the evolution of the particle field in time and space can be predicted by the equations if the initial distribution of density field is given. For ease of reference, Table 3 lists the quantities and equations of the particle field.

Table 3. The quantities and equations of particle field

\begin{tabular}{ccc}
\hline Name & Definition and Equation & Scale \\
\hline Mass density & $\rho=\rho(\boldsymbol{x}, t)$ & $\rho_{s}=\frac{M_{s}}{V_{s}}$ \\
\hline Momentum density & $\boldsymbol{j}(\boldsymbol{x}, t)=\rho \boldsymbol{u}$ & $j_{s}=\frac{M_{s} u_{s}}{V_{s}}$ \\
\hline Velocity & $\boldsymbol{u}(\boldsymbol{x}, t)=\boldsymbol{j} / \rho$ & $u_{s}=c$ \\
\hline Mass conservation & $\frac{\partial \rho}{\partial t}+\nabla \cdot \boldsymbol{j}=0$ & $\frac{\rho_{s}}{t_{s}}=\frac{M_{s}}{V_{s} t_{s}}$ \\
\hline Mass potential & $\Phi(\boldsymbol{x}, t)=\frac{-1}{4 \pi \varphi_{s}} \iiint_{V} \frac{\rho\left(\boldsymbol{x}^{\prime}, t\right)}{\left|\boldsymbol{x}-\boldsymbol{x}^{\prime}\right|} d \boldsymbol{x}^{\prime}$ & $\Phi_{s}=\frac{J_{s}}{M_{s}}=u_{s}^{2}$ \\
\hline Momentum potential & $\boldsymbol{A}(\boldsymbol{x}, t)=\frac{\alpha_{s}}{4 \pi} \iiint_{V} \frac{\boldsymbol{j}\left(\boldsymbol{x}^{\prime}, t\right)}{\left|\boldsymbol{x}-\boldsymbol{x}^{\prime}\right|} d \boldsymbol{x}^{\prime}$ & $A_{s}=\frac{K_{s}}{p_{s}}=u_{s}$ \\
\hline Total potential energy & $J(t)=\iiint_{V} \rho\left(\boldsymbol{x}^{\prime}, t\right) \Phi\left(\boldsymbol{x}^{\prime}, t\right) d \boldsymbol{x}^{\prime}$ & $J_{s}=M_{s} u_{s}^{2}$ \\
\hline$K(t)=\iiint_{V} \boldsymbol{j}\left(\boldsymbol{x}^{\prime}, t\right) \cdot \boldsymbol{A}\left(\boldsymbol{x}^{\prime}, t\right) d \boldsymbol{x}^{\prime}$ & $K_{s}=M_{s} u_{s}^{2}$
\end{tabular}




\begin{tabular}{|c|c|c|}
\hline Medium constant & $\varphi=\tilde{\varphi} \cdot \varphi_{s} ; \quad \tilde{\varphi}=4 \pi$ & $\varphi_{s}=\frac{M_{s}}{r_{s} \Phi_{s}}=\frac{M_{s}}{r_{s} u_{s}^{2}}$ \\
\hline Dynamic constant & $\alpha=\tilde{\alpha} \cdot \alpha_{s} ; \quad \tilde{\alpha}=(4 \pi)^{-1}$ & $\alpha_{s}=\frac{A_{s} r_{s}}{p_{s}}=\frac{r_{s}}{M_{s}}$ \\
\hline Boundary condition & $B(S)=\frac{-\alpha_{S}}{4 \pi} \oiint_{S} \frac{\boldsymbol{j}\left(\boldsymbol{x}^{\prime}, t\right) \cdot d \boldsymbol{S}^{\prime}}{r}=0$ & $\alpha_{s} j_{s} r_{s}=\frac{1}{t_{s}}$ \\
\hline $\begin{array}{c}\text { Gradient field } \\
\text { (Negative gradient of mass potential) }\end{array}$ & $\boldsymbol{G}=-\nabla \Phi=\frac{-1}{4 \pi \varphi_{s}} \iiint_{V} \frac{\rho\left(\boldsymbol{x}^{\prime}, t\right) \boldsymbol{r}}{r^{3}} d \boldsymbol{x}^{\prime}$ & $G_{s}=\frac{\Phi_{s}}{r_{s}}=\frac{r_{s}}{t_{s}^{2}}$ \\
\hline Curl of the gradient field & $\nabla \times \boldsymbol{G}=-\nabla \times \nabla \Phi \equiv 0$ & $\frac{G_{s}}{r_{s}}=\frac{1}{t_{s}^{2}}$ \\
\hline $\begin{array}{c}\text { Divergence of the gradient field } \\
\text { (Poisson equation of the mass potential) }\end{array}$ & $\nabla \cdot \boldsymbol{G}=-\nabla^{2} \Phi=-\rho / \varphi_{s}$ & $\frac{G_{s}}{r_{s}}=\frac{1}{t_{s}^{2}}$ \\
\hline Poisson equation of the gradient field & $\nabla^{2} \boldsymbol{G}=-\nabla \rho / \varphi_{s}$ & $\frac{G_{s}}{r_{s}^{2}}=\frac{1}{r_{s} t_{s}^{2}}$ \\
\hline $\begin{array}{c}\text { Divergence field, Wave equation } \\
\text { (Divergence of the momentum potential) }\end{array}$ & $D=\nabla \cdot \boldsymbol{A}=\frac{1}{u_{s}^{2}} \frac{\partial \Phi}{\partial t}$ & $D_{s}=\frac{A_{s}}{r_{s}}=\frac{1}{t_{s}}$ \\
\hline $\begin{array}{c}\text { Wave potential } \\
\text { (Solution of the wave equation) }\end{array}$ & $\begin{array}{c}\Phi_{W}=-W(\xi) ; \boldsymbol{A}_{W}=(\boldsymbol{\kappa} / \omega) W(\xi) \\
\xi=\boldsymbol{\kappa} \cdot \boldsymbol{x}-\omega t\end{array}$ & $\begin{array}{c}\Phi_{s}=u_{s}^{2} ; A_{s}=u_{s} \\
\xi_{s}=1\end{array}$ \\
\hline Gradient of the divergence field & $\nabla D=\nabla(\nabla \cdot \boldsymbol{A})=-\frac{1}{u_{s}^{2}} \frac{\partial \boldsymbol{G}}{\partial t}$ & $\frac{D_{s}}{r_{s}}=\frac{1}{r_{s} t_{s}}$ \\
\hline $\begin{array}{c}\text { Curl field } \\
\text { (Curl of the momentum potential) }\end{array}$ & $\boldsymbol{C}=\nabla \times \boldsymbol{A}=\frac{\alpha_{s}}{4 \pi} \iiint_{V} \frac{\boldsymbol{j}\left(\boldsymbol{x}^{\prime}, t\right) \times \boldsymbol{r}}{r^{3}} d \boldsymbol{x}^{\prime}$ & $C_{s}=\frac{A_{s}}{r_{s}}=\frac{1}{t_{s}}$ \\
\hline Divergence of the curl field & $\nabla \cdot \boldsymbol{C}=\nabla \cdot(\nabla \times \boldsymbol{A}) \equiv 0$ & $\frac{C_{s}}{r_{s}}=\frac{1}{r_{s} t_{s}}$ \\
\hline Curl of the curl field & $\nabla \times \boldsymbol{C}=\alpha_{s} \boldsymbol{j}-\frac{1}{u_{s}^{2}} \frac{\partial \boldsymbol{G}}{\partial t}$ & $\frac{C_{s}}{r_{s}}=\frac{1}{r_{s} t_{s}}$ \\
\hline $\begin{array}{c}\text { Poisson equation of } \\
\text { the momentum potential }\end{array}$ & $\nabla^{2} \boldsymbol{A}=-\alpha_{s} \boldsymbol{j}$ & $\frac{A_{s}}{r_{s}^{2}}=\frac{1}{r_{s} t_{s}}$ \\
\hline Poisson equation of the curl field & $\nabla^{2} \boldsymbol{C}=-\alpha_{s} \nabla \times \boldsymbol{j}$ & $\frac{C_{s}}{r_{s}^{2}}=\frac{1}{r_{s}^{2} t_{s}}$ \\
\hline
\end{tabular}

\section{$4 \quad$ Nature of Interactions}

\subsection{Forces and Motional Equation}

\subsubsection{Force definition}

The momentum of a gridon is not conserved due to the interaction between gridons. We define the force as the total derivative of momentum density. It can be directly obtained by quoting the equation (55b) as

As the divergence of dyad $\boldsymbol{j u}$ is

$$
\boldsymbol{f} \equiv \frac{D \boldsymbol{j}}{D t}=\frac{\partial \boldsymbol{j}}{\partial t}+\nabla \cdot(\boldsymbol{j u}) ; \quad f_{s}=\frac{j_{s}}{t_{s}}=\frac{\rho_{s} r_{s}}{t_{s}^{2}} .
$$

$$
\nabla \cdot(\boldsymbol{j} \boldsymbol{u})=(\nabla \cdot \boldsymbol{j}) \boldsymbol{u}+(\boldsymbol{j} \cdot \nabla) \boldsymbol{u}
$$

we can calculate the force by using Eqs. (57) and (81),

$$
\begin{aligned}
& \boldsymbol{f}=\frac{\partial \boldsymbol{j}}{\partial t}+\nabla \cdot(\boldsymbol{j u})=\left(\frac{\partial \rho}{\partial t} \boldsymbol{u}+\rho \frac{\partial \boldsymbol{u}}{\partial t}\right)+(\nabla \cdot \boldsymbol{j}) \boldsymbol{u}+(\boldsymbol{j} \cdot \nabla) \boldsymbol{u} \\
& =\rho \frac{\partial \boldsymbol{u}}{\partial t}+(\boldsymbol{j} \cdot \nabla) \boldsymbol{u}=\rho\left[\frac{\partial \boldsymbol{u}}{\partial t}+(\boldsymbol{u} \cdot \nabla) \boldsymbol{u}\right] .
\end{aligned}
$$

It can be written in the form of Newton second law

$$
\boldsymbol{f}=\rho \boldsymbol{a}, \quad \boldsymbol{a}=\frac{\partial \boldsymbol{u}}{\partial t}+(\boldsymbol{u} \cdot \nabla) \boldsymbol{u} ; \quad a_{s}=\frac{f_{s}}{\rho_{s}}=\frac{r_{s}}{t_{s}^{2}} .
$$


where $\boldsymbol{a}$ is the acceleration. The term $\partial \boldsymbol{u} / \partial t$ is linear acceleration and the term $(\boldsymbol{u} \cdot \nabla) \boldsymbol{u}$ is curvilinear acceleration. The acceleration so obtained has the same form as the material derivative of the velocity in fluid mechanics [12].

\subsubsection{Action force}

The action force comes from the coupling of density field and action field. The action force includes gradient force, divergence force and curl force.

(a) Gradient force. A gridon with mass density $\rho$ is subjected to a gradient force $\boldsymbol{f}_{G}$ in the gradient field $\boldsymbol{G}$.

$$
\boldsymbol{f}_{G}=\rho \boldsymbol{G}=-\rho \nabla \Phi ; \quad f_{s}=\rho_{s} G_{s}=\rho_{s} r_{s} / t_{s}^{2} .
$$

Since $\nabla \Phi$ could be positive or negative, the gradient force could be either attractive or repulsive. $\boldsymbol{f}_{G}$ is essentially a diffusion force caused by uneven mass distribution.

(b) Divergence force. A gridon with momentum density $\boldsymbol{j}$ is subjected to a divergence force $\boldsymbol{f}_{D}$ in the divergence field $D$.

$$
\boldsymbol{f}_{D}=D \boldsymbol{j}=\rho D \boldsymbol{u} ; \quad f_{s}=\rho_{s} D_{s} u_{s}=\rho_{s} r_{s} / t_{s}^{2} .
$$

The divergence force is parallel to the direction of velocity $\boldsymbol{u}$. Since $D=\nabla \cdot \boldsymbol{A}$ could be either negative or positive, the divergence force could be either resistance or propulsion. $\boldsymbol{f}_{D}$ is the force that an object is subjected to when it is vibrating and moving.

(c) Curl force. A gridon with momentum density $\boldsymbol{j}$ is subjected to a curl force $\boldsymbol{f}_{C}$ in the curl field $\boldsymbol{C}$.

$$
\boldsymbol{f}_{C}=\boldsymbol{C} \times \boldsymbol{j}=\rho \boldsymbol{C} \times \boldsymbol{u} ; f_{s}=\rho_{s} C_{s} u_{s}=\rho_{s} r_{s} / t_{s}^{2} .
$$

The curl force is perpendicular to the curl field and the moving direction. $\boldsymbol{f}_{C}$ is the force that an object is subjected to when it is spinning and moving.

\subsubsection{Motion equation}

The action force is the interaction of one gridon with all other gridons, which is just the reason causing the change of gridon momentum. Let $\boldsymbol{f}=\boldsymbol{f}_{G}+\boldsymbol{f}_{D}+\boldsymbol{f}_{C}$, we obtain an equation of motion for any object

$$
\boldsymbol{f}=\rho\left[\frac{\partial \boldsymbol{u}}{\partial t}+(\boldsymbol{u} \cdot \nabla) \boldsymbol{u}\right]=\rho(\boldsymbol{G}+D \boldsymbol{u}+\boldsymbol{C} \times \boldsymbol{u}) .
$$

This is a formula in the place of Navier-Stokes equation [12]. It is applicable to a gridon in continuous field and is also applicable to any individual object. For a system of momentum conservation, $\boldsymbol{f}=0$, the object undergoes a free motion.

\subsection{Gravitational Force}

\subsubsection{Origin of gravitation}

It is known that the Newtonian gravitational potential can be expressed as

$$
\Phi_{\mathrm{g}}(\boldsymbol{x}, t)=-\gamma \iiint_{V} \frac{\rho\left(\boldsymbol{x}^{\prime}, t\right)}{\left|\boldsymbol{x}-\boldsymbol{x}^{\prime}\right|} d \boldsymbol{x}^{\prime}
$$

where $\gamma=6.6742867 \times 10^{-11} \mathrm{Nm}^{2} \mathrm{~kg}^{-2}$ is the gravitational constant. It is seen that the Newtonian gravitational potential and the mass potential are completely equivalent. Mass potential is a scalar potential, the corresponding vector potential is the momentum potential. Therefore, the set of particle field equations is essentially the gravitational field equations in three-dimensional flat space, instead of that in four-dimensional curved spacetime. The property of gravitation can be analyzed according to the theory of particle field.

The medium constant and the dynamic constant are determined by the gravitational constant with the signal speed $u_{s}=c=2.9979246 \times 10^{8} \mathrm{~ms}^{-1}$.

$$
\begin{gathered}
\varphi=\gamma^{-1}=1.4982874 \times 10^{10} \mathrm{~N}^{-1} \mathrm{~m}^{-2} \mathrm{~kg}^{2}, \\
\alpha=\varphi^{-1} \mathrm{c}^{-2}=\gamma c^{-2}=7.4261454 \times 10^{-28} \mathrm{Ns}^{2} \mathrm{~kg}^{-2} .
\end{gathered}
$$

The theory of particle field requires that space must be filled with particles, and there is no absolutely empty space (so-called vacuum). Since the primary particles are only the proton and the electron, the vacuum must be filled with pure electrons. Therefore, the electrons in cosmic background are just the so-called dark matter.

\subsubsection{Properties of gravitation}

According to Eq. (84), an object is subjected to a gradient force depending on the gradient of mass potential. If $\nabla \Phi>0$, the acceleration is negative. If $\nabla \Phi<0$, the acceleration is positive. Therefore, the 
gravitation can be either attractive or repulsive. In this sense, the term "gravitation" is not limited to attraction. The repulsive force roots from the effect of volume repulsion.

According to Eq. (85), a moving object is subjected to a force proportional to the divergence field $D$. If $D<0$, it is a resistance. If $D>0$, it is a propulsion. Divergence force exists when the object is both moving and vibrating. For example, a vibrating baseball in flight is subjected to the divergence force.

According to Eq. (86), a moving object is subjected to a curl force $\boldsymbol{C} \times \boldsymbol{j}$, which is perpendicular to the curl field and the moving direction. Curl force exists when the object is both moving and spinning. For example, a spinning disc in flight is subjected to the curl force.

The general motion of an object follows the equation of motion. According to Eq. (87), a system in momentum conservation undergoes a free motion. For example, the earth and the moon orbiting the earth can be considered approximately as a system in free motion.

\subsubsection{Gravitational wave}

Eq. (64) predicts the particle wave with wave potential Eq. (67). In essence, all waves are particle waves, and gravitational waves are no exception. Waves in common media (solids, liquids and gases) are molecular waves that travel at the speed of sound. Waves in pure electron system (cosmic background) are electron waves that travel at the speed of light. Electrons are the medium of gravitation, and are the so-called gravitons. The propagation of gravitational waves is limited at a speed $c$. Gravitation is not an instantaneous interaction.

\subsection{Electromagnetic Force}

\subsubsection{Origin of electromagnetism}

Let $\rho_{e}$ be the density of electric charge and $\boldsymbol{j}_{e}$ be the density of electric current, the electric potential $\Phi_{e}$ and magnetic potential $\boldsymbol{A}_{e}$ are known as

$$
\begin{gathered}
\Phi_{e}(\boldsymbol{x}, t)=\frac{-1}{\epsilon} \iiint_{V} \frac{\rho_{e}\left(\boldsymbol{x}^{\prime}, t\right)}{\left|\boldsymbol{x}-\boldsymbol{x}^{\prime}\right|} d \boldsymbol{x}^{\prime} ; \quad \boldsymbol{A}_{e}(\boldsymbol{x}, t)=\mu \iiint_{V} \frac{\boldsymbol{j}_{e}\left(\boldsymbol{x}^{\prime}, t\right)}{\left|\boldsymbol{x}-\boldsymbol{x}^{\prime}\right|} d \boldsymbol{x}^{\prime} \\
\epsilon=4 \pi \epsilon_{s} ; \quad \mu=(4 \pi)^{-1} \mu_{s} ; \quad \epsilon \mu=\epsilon_{s} \mu_{s}=c^{2},
\end{gathered}
$$

where $\epsilon_{s}=8.8541877 \times 10^{-12} \mathrm{C}^{2} \mathrm{~N}^{-1} \mathrm{~m}^{-2}$ is the vacuum permittivity and $\mu_{s}=4 \pi \times 10^{-7} \mathrm{NC}^{-2} \mathrm{~s}^{2}$ is the vacuum permeability.

By introducing a scale factor $\theta$ as

$$
\theta=\frac{\epsilon}{\varphi}=\frac{\alpha}{\mu}=\frac{\epsilon_{s}}{\varphi_{s}}=\frac{\alpha_{s}}{\mu_{s}},
$$

we can calculate $\theta$ with the gravitational constant and the vacuum permittivity.

$$
\theta=\epsilon / \varphi=4 \pi \epsilon_{s} \gamma=7.4261454 \times 10^{-21} \mathrm{C}^{2} \mathrm{~kg}^{-2} ; \theta_{s}=Q_{s}^{2} / M_{s}^{2}
$$

It is known that an electron has the mass $M_{e}=9.1093821 \times 10^{-31} \mathrm{~kg}$ and the electric charge $Q_{e}=1.6021765 \times 10^{-19} \mathrm{C}$. It gives an equivalent relation of the mass to charge,

$$
\beta=M_{e} / Q_{e}=5.6856296 \times 10^{-12} \mathrm{~kg} \mathrm{C}^{-1} ; \quad \beta_{s}=M_{s} / Q_{s} .
$$

Denoting $\boldsymbol{E}_{\boldsymbol{e}}$ as the electric field, $\boldsymbol{B}_{\boldsymbol{e}}$ as the magnetic induction, it is easy to show that the electromagnetic field is equivalent to the particle field under following transformations.

$$
\begin{gathered}
\rho_{e}=\beta \theta \rho=\left(4.2222312 \times 10^{-32} \mathrm{Ckg}^{-1}\right) \rho ; \\
\boldsymbol{j}_{e}=\beta \rho \boldsymbol{j}=\left(4.2222312 \times 10^{-32} \mathrm{Ckg}^{-1}\right) \boldsymbol{j} \\
\epsilon_{s}=\theta \varphi_{s}=\left(7.4261454 \times 10^{-21} \mathrm{C}^{2} \mathrm{~kg}^{-2}\right) \varphi_{s} ; \\
\mu_{s}=\theta^{-1} \alpha_{s}=\left(1.3465936 \times 10^{20} \mathrm{C}^{-2} \mathrm{~kg}^{2}\right) \alpha_{s} \\
\Phi_{e}=\beta \Phi=\left(5.6856296 \times 10^{-12} \mathrm{kgC}^{-1}\right) \Phi \\
\boldsymbol{A}_{e}=\beta \boldsymbol{A}=\left(5.6856296 \times 10^{-12} \mathrm{kgC}^{-1}\right) \boldsymbol{A} \\
\boldsymbol{E}_{e}=\beta \boldsymbol{G}=\left(5.6856296 \times 10^{-12} \mathrm{kgC}^{-1}\right) \boldsymbol{G} \\
\boldsymbol{B}_{e}=\beta \boldsymbol{C}=\left(5.6856296 \times 10^{-12} \mathrm{kgC}^{-1}\right) \boldsymbol{C} .
\end{gathered}
$$

It is clear that both electromagnetic field and gravitational field originate from the interaction of particles.

\subsubsection{Limitation of electrodynamics}

According to the transformation relations (95)-(98), we obtain four equations that are similar to the Maxwell equations,

$$
\nabla \cdot \boldsymbol{E}_{e}=-\frac{\rho_{e}}{\epsilon_{s}} ; \nabla \cdot \boldsymbol{B}_{e}=0 ; \nabla \times \boldsymbol{E}_{e}=0 ; \nabla \times \boldsymbol{B}_{e}=\mu_{s} \boldsymbol{j}_{e}-\mu_{s} \epsilon_{s} \frac{\partial \boldsymbol{E}_{e}}{\partial t} .
$$


Compared with Maxwell equations, the main difference can be found in the third equation. Electromagnetic field theory assumes that the curl of electric field is non-zero, while particle field theory requires that the curl of gradient field must be zero. Obviously, the Maxwell equations are not complete, because Eq. (99) is only a part of the particle field equations. The complete field equations contain the second-order space derivative of the action field.

In particle field theory, both the mass potential and the momentum potential have clear physical meanings. They cannot be added with any constants. In the electromagnetic field theory, however, the values of electric potential and magnetic potential have no absolute meanings, so the electromagnetic field has the freedom of gauge transformation. This is an important difference between particle field theory and electromagnetic field theory.

With Lorentz gauge, the electric and magnetic potentials satisfy the D'Alembert equations [13], i.e.

$$
\nabla^{2} \Phi_{e}-\frac{1}{c^{2}} \frac{\partial^{2} \Phi_{e}}{\partial t^{2}}=-\frac{\rho_{e}}{\epsilon_{s}} ; \quad \nabla^{2} \boldsymbol{A}_{e}-\frac{1}{c^{2}} \frac{\partial^{2} \boldsymbol{A}_{e}}{\partial t^{2}}=-\mu_{s} \boldsymbol{j}_{e}
$$

It is an inhomogeneous wave equation containing the second-order time derivative of the potentials, and its solution is in the complex domain. In particle field theory, however, divergence field represents the wave equation containing only the first-order time derivative of the mass potential. The solution of divergence field is the wave potential in the domain of real number. It can be said that the essence of light wave is electronic wave. In other words, electrons are the medium for transmitting light waves. Therefore, electron and photon is identical particle.

Above analysis concludes that electrons are the medium for transmitting both light waves and gravitational waves in the background of the universe. Photon and graviton are the same entity as electron, also, dark matter. Electronic waves travel at the speed of light. The common substance is a molecular system, and the wave speed in common substance is the sound speed. With sound speed as the signal speed, the particle field equations are applicable to general substances.

\subsubsection{Stability of atomic structure}

Eq. (95) clearly shows that the electric charge is equivalent to the mass, and the electric current is equivalent to the momentum. In the particle field theory, the proton and electron have only mass and volume. As the mass is always positive, the attraction or repulsion between proton and electron depends on the positive or negative gradient fields. This feature ensures that a proton and an electron can form stable neutron, just like the case of earth-moon system. The weak force that binds proton and electron to form neutron is a combination of three action (gradient, divergence and curl) forces. For the same reason, the strong force that binds protons and neutrons to form a nucleus is also a combination of the three action forces. The range of strong interaction is $10^{-15} \mathrm{~m}$, indicating that the size of the proton is approximately the same as this value.

One defect of electromagnetic theory is that it does not contain the repulsion between proton and electron. So, proton and electron cannot constitute a stable neutron unless the weak force is assumed. Another defect of electromagnetic theory is that it does not contain the attraction between protons. So, protons and neutrons cannot constitute a stable nucleus unless the strong force is assumed. In the case of point particles, electron and proton will be infinitely close to each other under electric attraction. Therefore, the system of protons and electrons cannot form a stable atom in classical electrodynamics.

The study of the stability of atomic structures spawned the birth of quantum mechanics. Quantum theory has its inherent limitations as it does not find the origins of gravitation and electromagnetism. By the introduction of volume repulsion, the interaction of particles is unified in the real physics. The universality of real physical theory also roots from the principle of objectivity, because the field equations have origin irrelevance and scale irrelevance.

\section{Summary}

The real physical theory is based on the model of body particle and the viewpoint of real space. The attributes of body particles are only mass and volume, and any object structure can be interpreted as the spatial nesting of protons and electrons. Scale is an important concept that not only provides a scheme for digitalized physics, but also produces a criterion for universal physics. Objectivity is a fundamental principle that excludes all subjective factors from physics. The origin irrelevance guarantees the translation invariance of space and time, and the scale irrelevance guarantees the measurement invariance under scale 
transformation.

Starting from the finite particle density, we construct a scalar potential and a vector potential, and then derives a complete set of particle field equations. The particle field equations are actually a set of universal field equations in three-dimensional flat space, instead of that in four-dimensional curved space-time. It is applied to gravitational field, electromagnetic field and classical flow field. It is shown that gravitation includes both attraction and repulsion, depending on the negative or positive mass gradient. The electric charge is equivalent to mass, and electric current is equivalent to momentum. The sign of electric charge actually comes from the positive or negative of the mass gradient. The gravitation and electromagnetism both originate from the interaction of body particles. The common media (gas, liquid and solid) are molecular fields, and vacuum is actually a pure electron field. The particle field theory predicts the waves propagating at a signal speed in particle medium, indicating that the essence of sound waves, light waves and gravitational waves are all particle waves. If photon and graviton are regarded as body particles identical to electron, then the electrons are the dark matter in the background of the universe. In this way, all physical fields and interactions are unified within a formal axiomatic system of the particle field theory.

Acknowledgments. This research is partially supported by NSFC (No.61775102). The author sincerely thanks Professor Francis T. S. Yu for his strong support and inspiring discussion. The author also thanks Mr. Ning Tian for his enthusiastic assistance in many ways.

\section{References}

1. I. Newton. Mathematical Principles of Natural Philosophy. Daniel Adee Press, New York. 1846.

2. J. C. Maxwell. "A dynamical theory of the electromagnetic field". Philosophical Transactions of the Royal Society of London. 155, 459-512. 1865. doi:10.1098/rstl.1865.0008.

3. A. Einstein. "Zur Elektrodynamik bewegter Körper". Annalen der Physik, 17, 891. 1905.

4. R. M. Wald. General Relativity .University of Chicago Press, Chicago. 1984.

5. S. Braibant, G. Giacomelli, and M. Spurio. Particles and Fundamental Interactions: An Introduction to Particle Physics. Springer Netherlands. 2012.

6. C. Rovelli. "Quantum gravity", Scholarpedia, 3(5), 7117. 2008.

7. B. Zwiebach. A First Course in String Theory. Cambridge University Press, Cambrige. 2004.

8. X. L. Zuo, W.J. Li and Y.C. Liu. Discrete mathematics, Shanghai Scientific and Technological Literature Press, Shanghai. 1982.

9. P. Smith, An Introduction to Gödel's Theorems. Cambridge University Press, Cambrige. 2007.

10. Z. C. Liang. Physical principles of finite particle system. Scientific Research Publishing, Wuhan. 2015. http://www.scirp.org/book/DetailedInforOfABook.aspx?bookID=2333.

11. Z. C. Liang. "Essence of light: particle, field and interaction". in Proc. SPIE (Optics+Photonics 2018, San Diego), 10755, 1075501-10755014. doi:10.1117/12.2316422.

12. S. L. Jin and Y.L. Ma. Theoretical Mechanics. Higher Education Press, Beijing. 1985.

13. S. H. Guo. Electrodynamics, Higher Education Press, Beijing. 2008.

14. K. M. Liang. Methods of mathematical physics. Higher Education Press, Beijing. 2010. 